\title{
A SUFFICIENT CONDITION FOR $k$-PATH HAMILTONIAN DIGRAPHS
}

BY JOHN ROBERTS

Communicated by Walter Gautschi, September 7, 1975

A directed graph (or digraph) $D$ is: (1) traceable if $D$ has a hamiltonian path; (2) hamiltonian if $D$ has a hamiltonian cycle; (3) strongly hamiltonian if $D$ has arcs and each arc lies on a hamiltonian cycle; (4) hamiltonian-connected if $D$ has a hamiltonian $u-v$ path for every pair of distinct vertices $u$ and $v$; (5) $k$-path traceable if every path of length not exceeding $k$ is contained in a hamiltonian path; and (6) $k$-path hamiltonian if every path of length not exceeding $k$ is contained in a hamiltonian cycle.

The indegree and the outdegree of a vertex $v$ are denoted by $\operatorname{id}(v)$ and od( $(v)$ respectively. A digraph $D$ of order $p$ is of Ore-type $(k)$ if $\operatorname{od}(u)+\operatorname{id}(v) \geqslant p+k$ whenever $u$ and $v$ are distinct vertices for which $u v$ is not an $\operatorname{arc}$ of $D$.

In this research announcement we outline a proof of the following result, a complete proof of which will appear elsewhere, and present some consequences of it.

THEOREM. If a nontrivial digraph $D$ is of Ore-type ( $k$ ), $k \geqslant 0$, then $D$ is k-path hamiltonian.

Proof. Let $D$ have order $p \geqslant 2$. First, observe that $D$ is strong. Since the result holds if $D$ is the complete symmetric digraph $K_{p}$, we assume that $D \neq K_{p}$. This in turn implies that $p \geqslant k+4$. Also, it can be shown that every path of length not exceeding $k$ is contained in a path of length $(k+1)$ and this longer path is contained in a cycle.

Suppose $D$ has a path $P: v_{1}, v_{2}, \ldots, v_{k+1}$ of length $k$ which is contained in no hamiltonian cycle. Let $C: v_{1}, v_{2}, \ldots, v_{n}, v_{1}$ be any longest cycle containing $P$. Then, $V \equiv V(D)-V(C) \neq \varnothing$, where $V(D)$ and $V(C)$ denote the vertex sets of $D$ and $C$ respectively.

Now, assume that $V$ has distinct vertices $u$ and $v$ for which $u v \notin E(D)$ and the subdigraph $\langle V\rangle$ induced by $V$ has no $v-u$ path. Then, $v u \notin E(D)$ implies that

$$
p+k \leqslant \operatorname{od}(v)+\operatorname{id}(u) \leqslant p-n-2+\operatorname{od}(v, C)+\operatorname{id}(u, C)
$$

where $\operatorname{od}(v, C)$ and $\operatorname{id}(u, C)$ denote the number of vertices in $C$ which are

AMS (MOS) subject classifications (1970). Primary $05 C 20$.

Key words and phrases. Digraphs, traceable, hamiltonian, hamiltonian-connected, strongly hamiltonian, $k$-path hamiltonian, $k$-path traceable. 
dominated by $v$ and dominate $u$, respectively. Then (1) implies that $n+k+2$ $\leqslant \operatorname{od}(v, C)+\mathrm{id}(u, C)$ and this implies that $\langle V\rangle$ has no $u \cdot v$ path. For suppose that $\langle V\rangle$ has such a path. Since $C$ is a longest cycle containing $P$, the digraph $D$ cannot contain both of the arcs $v_{i} u$ and $v v_{i+1}$ for $k+1 \leqslant i \leqslant n$. But this implies that $\operatorname{id}(u, C)+\operatorname{od}(v, C) \leqslant n+k$ and this is a contradiction. Using the fact that $u v \notin E(D)$, we obtain

$$
p+k \leqslant \operatorname{od}(u)+\operatorname{id}(v) \leqslant p-n-2+\operatorname{od}(u, C)+\operatorname{id}(v, C)
$$

which also implies that $n+k+2 \leqslant \operatorname{od}(u, C)+\operatorname{id}(v, C)$. Together with the preceding result, this implies that either

$$
n+k+2 \leqslant \operatorname{od}(u, C)+\mathrm{id}(u, C) \text { or } n+k+2 \leqslant \operatorname{od}(v, C)+\mathrm{id}(v, C) .
$$

In either case, it follows that $D$ has a longer cycle containing $P$ which is impossible. Thus, for distinct vertices $u$ and $v$ of $\langle V\rangle$, either $u v \in E(\langle V\rangle)$ or $\langle V\rangle$ has a $v-u$ path. If $\langle V\rangle$ has a $v-u$ path, then $\operatorname{od}(u, C)+\operatorname{id}(v, C) \leqslant n+k$. Thus,

$$
\operatorname{od}(u,\langle V\rangle)+\operatorname{id}(v,\langle V\rangle) \geqslant p-n=|V|
$$

whenever $u \neq v$ and $u v \notin E(\langle V\rangle)$. Hence, $\langle V\rangle$ is strongly connected.

Let $W$ be the subpath $v_{k+1}, v_{k+2}, \ldots, v_{n}, v_{n+1}=v_{1}$ of $C$. Since $n \geqslant$ $k+2$, the path $W$ has order at least 3 ; in fact $W$ has at least 3 vertices which are dominated by vertices of $V$ and at least 3 vertices which dominate vertices of $V$. It now suffices to consider the following two cases: (i) the path $W$ has a nontrivial subpath $W^{\prime}$ whose initial vertex dominates a vertex of $V$ and whose terminal vertex is dominated by a vertex of $V$; and (ii) the path $W$ has no such subpath. Since consideration of either case leads to contradiction, our assumption that $V \neq \varnothing$ must be false. Hence, $C$ is a hamiltonian cycle and the theorem follows.

Let $m, n \geqslant 1$ and $k \geqslant 0$. The symmetric join $K_{k+2}+\left(K_{m} \cup K_{n}\right)$ of $K_{k+2}$ and the disjoint union of $K_{m}$ and $K_{n}$ is an Ore-type (k) digraph which is not $(k+1)$-path hamiltonian. Hence, the result is "best possible."

The preceding result generalizes several results from graph theory and digraph theory, which we present below.

Corollary. If the digraph $D$ is of Ore-type $(k), k \geqslant-1$, then $D$ is $(k+1)$-path traceable.

COROllary (WoOdall [5]). If a nontrivial digraph is of Ore-type (0), then it is hamiltonian.

COROLlaRY. If a nontrivial digraph is of Ore-type (1), then it is both strongly hamiltonian and hamiltonian-connected.

A (undirected) graph of order $p$ is of Ore-type $(k)$ if the sum of the degrees 
of nonadjacent vertices is at least $(p+k)$. By considering symmetric digraphs, we obtain the following results.

COROLlARY (ORE [3]). If a graph with order at least 3 is of Ore-type (0), then it is hamiltonian.

COROLlARY (ORE [4]). If a graph is of Ore-type (1), then it is hamiltonian-connected.

COROLlaRY (KRONK [2]). If a graph of order $p \geqslant 3$ is of Ore-type (k), $k \geqslant 0$, then it is $k$-path hamiltonian.

COROLlARY (KAPOOR AND THECKEDATH [1]). If a graph is of Ore-type $(k), k \geqslant-1$, then it is $(k+1)$-path traceable.

\section{REFERENCES}

1. S. F. Kapoor and K. K. Theckedath, Highly traceable graphs, Sankhyā Ser. A 33 (1971), 211-216. MR 47 \#3254.

2. H. V. Kronk, A note on k-path Hamiltonian graphs, J. Combinatorial Theory 7 (1969), 104-106. MR 39 \#6772. \#9454.

3. O. Ore, Note on Hamilton circuits, Amer. Math. Monthly 67 (1960), 55. MR 22

4. Hamilton connected graphs, J. Math. Pures Appl. (9) 42 (1963), 21-27. MR 26 \#4336.

5. D. R. Woodall, Sufficient conditions for circuits in graphs, Proc. London Math. Soc. (3) 24 (1972), 739-755. MR 47 \#6549.

DEPARTMENT OF MATHEMATICS, UNIVERSITY OF LOUISVILLE, LOUISVILLE, KENTUCKY 40208 